\title{
Fixed Subgroups In Free Groups: A Survey
}

\author{
E. Ventura
}

\begin{abstract}
This note is a survey of the main results known about fixed subgroups of endomorphisms of finitely generated free groups. A historic point of view is taken, emphasizing the evolution of this line of research, from its beginning to the present time. The article concludes with a section containing the main open problems and conjectures, with some comments and discussions on them.
\end{abstract}

\section{Introduction and notation}

The purpose of this note is to survey what is known about fixed subgroups of finitely generated free groups. Descriptions, comments and remarks on the proofs of known results will be presented instead of the proofs themselves, for which we refer the reader to the corresponding original papers. We will also take the opportunity to review the history of this particular topic in Group Theory, which goes back to the 1970's. However, it is not our intention to be exhaustive in this survey. We apologize to those authors who contributed to this line of research and are not cited here. In sections 2-7 we will adopt a historic point of view, describing the results chronologically as they appeared in the literature, even when some of them improve older ones. Finally, in section 8 , a list of some conjectures and open problems in this area of research will be provided and discussed.

The whole paper is about finitely generated free groups. From now on, $F_{n}$ will denote a free group of rank $n \geq 0$, while $F$ will be used to denote an arbitrary free group (possibly with infinite rank). It is well known that every subgroup of a free group is also free (see, for example, Theorem I.8.4 in [11]). But, in general, its rank can be larger than the rank of the ambient group. In fact, it is easy to see that the subgroup $H=\left\langle b^{-r} a b^{r}, r \in \mathbb{Z}\right\rangle$ of $F_{2}=\langle a, b\rangle$ is free of countably infinite rank. So, $F_{\aleph_{0}}$ is a subgroup of $F_{2}$. Therefore, every free group of finite or countably infinite rank can be viewed as a subgroup of every other, with the obvious exception of the trivial group, and of the free group with rank 1, which is the group of integers. Also, it is well known that the intersection of finitely generated subgroups of a free group is again finitely generated (see [30] or Theorem I.8.8 in [11]), while its rank can be of the order of the product of the two ranks. This behavior, which is completely different from what happens in other more classical algebraic contexts, motivated a lot of research dedicated to compute or bound the rank of several subgroups 
in different situations. We are interested in the case involving fixed subgroups of endomorphisms of $F_{n}$.

The reduced rank of a free group $F$, denoted $\tilde{r}(F)$, is $\max \{0, r(F)-1\}$, that is, one less than the rank, except for the trivial group where the reduced rank coincides with the rank, which is zero. So, a free group has reduced rank zero if and only if it is cyclic.

Let $\operatorname{End}\left(F_{n}\right)$ denote the monoid of endomorphisms of $F_{n}$, and $\operatorname{Aut}\left(F_{n}\right)$ the group of automorphisms of $F_{n}$ (so, $\operatorname{Aut}\left(F_{n}\right)$ is the group of units of $\operatorname{End}\left(F_{n}\right)$ ). Let $\operatorname{Inj}\left(F_{n}\right)$ denote the set of injective endomorphisms of $F_{n}$, a submonoid of $\operatorname{End}\left(F_{n}\right)$ containing $A u t\left(F_{n}\right)$. Finally, we denote by $\operatorname{Out}\left(F_{n}\right)$ the group of outer automorphisms of $F_{n}$, that is, $A u t\left(F_{n}\right)$ modulo the (normal) subgroup of conjugations, also called inner automorphisms. For each $u \in F_{n}$, we let $u$ again denote the corresponding right conjugation, $u: F_{n} \rightarrow F_{n}, x \mapsto x^{u}=u^{-1} x u$.

We let elements of $\operatorname{End}\left(F_{n}\right)$ act on the right of $F_{n}$ and, if there is no risk of confusion, we will omit the parenthesis for the argument. Thus, $x \phi$ denotes the image of $x$ under $\phi$, and $x \phi_{1} \phi_{2}$ denotes $\left(x \phi_{1}\right) \phi_{2}$.

For any $S \subseteq \operatorname{End}\left(F_{n}\right)$, let Fix $S$ denote the set consisting of the elements of $F_{n}$ which are fixed by every element of $S$ (with the convention that Fix $S=F_{n}$ when $S$ is empty). Then, Fix $S$ is a subgroup of $F_{n}$, called the fixed subgroup of $S$ or the subgroup fixed by $S$. When $S$ is a singleton, $S=\{\phi\}$, we simply write Fix $\phi$ instead of Fix $\{\phi\}$ for the fixed subgroup of $\phi$. So, Fix $S=\cap_{\phi \in S}$ Fix $\phi$. Clearly, if $S \subseteq \operatorname{End}\left(F_{n}\right)$ and $M$ is the submonoid of $\operatorname{End}\left(F_{n}\right)$ generated by $S$ then Fix $S=$ Fix $M$.

Following the notation introduced in [38], a subgroup $H \leq F_{n}$ is called endofixed if $H=$ Fix $S$ for some $S \subseteq \operatorname{End}\left(F_{n}\right)$. If $S$ can be chosen to lie in $\operatorname{Inj}\left(F_{n}\right)$ (resp. $\left.A u t\left(F_{n}\right)\right)$ we further say that $H$ is a mono-fixed (resp. auto-fixed) subgroup of $F_{n}$. And if $S$ can be chosen to be a singleton, $S=\{\phi\}$ for some $\phi \in \operatorname{End}\left(F_{n}\right)$ (resp. $\phi \in \operatorname{Inj}\left(F_{n}\right), \phi \in \operatorname{Aut}\left(F_{n}\right)$ ) we say more explicitly that $H$ is a 1 -endo-fixed (resp. 1-mono-fixed, 1-auto-fixed) subgroup of $F_{n}$.

Let $\phi \in \operatorname{End}\left(F_{n}\right)$. We will concentrate on those results concerning $\operatorname{Fix} \phi$ as a subgroup of $F_{n}$ and, for instance, only a few comments will be made about the nice recent results on infinite words fixed by the extension of $\phi$ to the boundary of $F_{n}$. Also, we will not be concerned in general about isolated fixed words, or about similar results in non-free groups.

The study of fixed subgroups in free groups began in 1975 with the paper [15] by J. Dyer and P. Scott. Since then, new results have been appearing constantly. It is agreed that the main and deepest result in this line of research is the BestvinaHandel Theorem, published in [2]. For this reason, the present paper is organized as follows. In section 2 we review the original motivations, as well as the first partial results. In section 3 we review the main results about fixed subgroups obtained in the period 1982-1992, before the Bestvina-Handel Theorem. Section 4 is entirely dedicated to the Bestvina-Handel theory. Section 5 is about the results obtained by using the Bestvina-Handel Theorem and by generalizing it. Section 6 considers the maximal rank case, while section 7 is dedicated to the concept of inertia, probably the strongest property currently known for fixed subgroups of finitely generated free groups. Finally, section 8 contains a list of conjectures and open problems in this line of research, as well as several comments and discussions. 


\section{The Scott conjecture}

In 1975, J. Dyer and P. Scott published the paper [15]. Using a theorem of Karrass-Pietrowski-Solitar about the structure of free-by-finite groups, the authors obtained the following result:

THEOREM 2.1 (Dyer-Scott, [15]). If $G$ is a finite group of automorphisms of a free group $F$, then Fix $G$ is a free factor of $F$. In particular, for every finite order automorphism $\phi \in \operatorname{Aut}\left(F_{n}\right)$, we have $r($ Fix $\phi) \leq n$.

This paper can be considered as the starting point of the line of research about fixed subgroups in finitely generated free groups. In [15], the authors mentioned that it was not known if Fix $G$ is finitely generated for arbitrary subgroups $G \leq$ $\operatorname{Aut}\left(F_{n}\right)$. In view of his result, P. Scott conjectured that this is the case at least for single automorphisms (i.e. when $G$ is cyclic). His main motivation was the following classical result due to J. Nielsen:

TheOREM 2.2 (Nielsen, [41]). Let $G_{p}$ be the fundamental group of a closed orientable surface with genus $p \geq 1$, and let $H$ be the subgroup fixed by some automorphism of $G_{p}$. Then, either $H=G_{p}$ or $H$ is free with rank at most $2 p-1$. Furthermore, if $H$ is not cyclic, then $G_{p}$ has a set of $2 p$ generators such that some subset of it generates $H$.

In 1975, no example was known of a 1-auto-fixed subgroup of $F_{n}$ with rank bigger than $n$. So, one could also conjecture that $r($ Fix $\phi) \leq n$ for every $\phi \in$ $\operatorname{Aut}\left(F_{n}\right)$. The finiteness of this rank or the previous inequality can also be studied for endomorphisms instead of automorphisms, and more generally, for arbitrary sets of endomorphisms. Soon, all these statements became generically referred to as the Scott conjecture. And time justified it, since it is now known that $r($ Fix $S) \leq n$ for every subset $S \subseteq \operatorname{End}\left(F_{n}\right)$.

In 1977, W. Jaco and P. B. Shalen published [34], where they studied the fixed and periodic subgroups of those automorphisms of the fundamental group of a compact connected surface $T$ induced by homeomorphisms of the surface $T$. Such an automorphism is called geometric for $T$. In the case where $T$ is closed and orientable, this provides an alternative and entirely different proof for Nielsen's Theorem (using the fact that, for these particular surfaces, every automorphism of $\pi_{1}(T)$ is geometric for $\left.T\right)$. For the cases where $T$ has boundary, the Jaco-Shalen Theorem gives the following corollary:

Theorem 2.3 (Jaco-Shalen, [34]). Let $\phi$ be an automorphism of $F_{n}$. If $\phi$ is geometric (for some compact connected surface), then Fix $\phi$ is either cyclic or a free factor of $F_{n}$. In particular, $r(\operatorname{Fix} \phi) \leq n$.

However, this result did not completely solve the Scott conjecture because, as J. Stallings showed in [44], there exist automorphisms of finitely generated free groups which are not geometric (for any compact connected surface). Concretely, J. Stallings introduced the concept of $\mathrm{PV}$-automorphism of $F_{n}: \phi \in \operatorname{Aut}\left(F_{n}\right)$ is a $P V$ automorphism when the absolute value of all the eigenvalues of its abelianization are less than 1, except exactly one which is larger than 1 . With a simple homological argument, he showed that no power of any PV-automorphism is geometric. The simplest such automorphism is the one in $F_{3}=\langle a, b, c\rangle$ given by $a \mapsto b, b \mapsto c$, $c \mapsto a b$. Furthermore, J. Stallings also conjectured that any PV-automorphism of 
$F_{n}$ with $n \geq 3$ has trivial fixed subgroup. This was known as the Stallings PVconjecture, and it provided new evidence in support of the Scott conjecture. As a motivation for his conjecture, J. Stallings proved (but did not write) that the fixed subgroup of a PV-automorphism of $F_{n}$ is contained in the third term in the lower central series of $F_{n}$ (see $\S 1$ in $\left.[46]\right)$.

The following step, in 1982, was the work [20] due to S. Gersten (and published in 1984). Here, the author considered another family of automorphisms of $F_{n}$, with motivations from one-dimensional geometry, i.e. from graphs. Let $F_{n}$ be viewed as the fundamental group of a bouquet $Y$ with $n$ petals. For every graph $X$ and every maximal subtree $T$ in $X$, one can collapse $T$ to a point and obtain $X / T$, which is a bouquet with as many petals as the rank of (the fundamental group of) $X$. There is also the natural projection map $X \rightarrow X / T$ inducing an isomorphism at the fundamental group level. An isomorphism $\phi$ of $F_{n}=\pi(Y, *)$ is called a change of maximal tree automorphism (CMT-automorphism for short) when there exists a (rank $n$ ) graph $X$, two maximal subtrees $T, T^{\prime}$ in $X$, identifications of $X / T$ and $X / T^{\prime}$ with $Y$, and a vertex $v$ in $X$ such that the corresponding isomorphisms $\eta_{T}: \pi(X, v) \rightarrow \pi(X / T, *) \simeq F$ and $\eta_{T^{\prime}}: \pi(X, v) \rightarrow \pi\left(X / T^{\prime}, *\right) \simeq F$ satisfy $\eta_{T}^{-1} \eta_{T^{\prime}}=$ $\phi$. In $[\mathbf{2 0}]$, S. Gersten solved the Scott conjecture for CMT-automorphisms proving the following result, using only combinatorial arguments:

THEOREM 2.4 (Gersten, [20]). Let $\phi$ be an automorphism of $F_{n}$. If $\phi$ is a CMT-automorphism, then $r($ Fix $\phi) \leq n$.

Despite being finite for every $n \geq 1$, the family of CMT-automorphisms of $F_{n}$ contains interesting elements. For example, all Whitehead automorphisms and all Squier's skew-Nielsen automorphisms of $F_{n}$ are CMT-automorphisms.

In addition, S. Gersten gave a method such that, when it terminates, it computes the fixed subgroup of a CMT-automorphism. However, this method is not really an algorithm because it can go into an infinite loop. The author analyzed some particular cases and provided the example given by the automorphism of $F_{n}=\left\langle x_{1}, \ldots, x_{n}\right\rangle, n \geq 2$, given by $x_{i} \mapsto x_{i+1} x_{1}$ for $i=1, \ldots, n-1$ and $x_{n} \mapsto x_{1}$, which is simultaneously a CMT-automorphism and a PV-automorphism, and has trivial fixed subgroup. This was the first infinite family of examples for which the PV-conjecture was known to be true.

\section{Fixed subgroups before Bestvina-Handel}

In the same year of its publication, an addendum to [20] appeared with more information. First, the author gave a simpler revised version of his method to compute fixed subgroups of CMT-automorphisms. Then, he announced a proof for the Scott conjecture in general. This proof was outlined in [21] but the details did not appear until 1987 in the paper [22]. The idea involves studying compositions of Whitehead automorphisms. These automorphisms generate $\operatorname{Aut}\left(F_{n}\right)$, and they are CMT-automorphisms so Gersten's method applies to them. After a careful combinatorial analysis, the author deduces that Fix $\phi$ is finitely generated for every $\phi \in \operatorname{Aut}\left(F_{n}\right)$. Then, using Howson's Theorem, the same can be said for finitely generated groups of automorphisms of $F_{n}$ :

THEOREM 3.1 (Gersten, [22]). Let $G$ be a finitely generated group of automorphism of $F_{n}$. Then, Fix $G$ is finitely generated. 
This solved the question originally asked by P. Scott for the finitely generated case. However, when one studies the composition of CMT-automorphisms, one loses control on the upper bound for the rank of the fixed subgroup, and the argument given by S. Gersten says only that this subgroup is finitely generated. In [22], an upper bound for $r($ Fix $\phi)$ is given, but it depends on the automorphism $\phi$ itself and can be arbitrarily large, in contrast with the uniform bound $r($ Fix $\phi) \leq n$ known at the time for periodic, geometric and CMT-automorphisms of $F_{n}$.

During the period 1982-1987, using completely different techniques, other authors obtained independent proofs of Gersten's Theorem, as well as several extensions of it. And due to the nuances of mathematical publication, many of Gersten's successors saw their work published before his paper appeared. For example, see $[\mathbf{2 4}],[\mathbf{2 5}],[\mathbf{2 6}],[\mathbf{1 0}]$ and $[\mathbf{4 6}]$, reviewed below.

In 1983, J. Stallings published [45], where some powerful graph-theoretical techniques, e.g. the pull-back of graphs, were first introduced and used to give alternative proofs for classical results on free groups. At the end of [45], the author ventured a generalization of the Scott conjecture, which has become known as the Stallings conjecture or the equalizer conjecture. Given two groups $G, G^{\prime}$, and two homomorphisms $\varphi, \phi: G \rightarrow G^{\prime}$, the equalizer of $\varphi$ and $\phi$, denoted $\operatorname{Eq}(\varphi, \phi)$, is the maximal subgroup of $G$ where both homomorphisms coincide,

$$
\operatorname{Eq}(\varphi, \phi)=\{x \in G \mid x \varphi=x \phi\} .
$$

The Stallings conjecture says that if $\varphi, \phi: F \rightarrow F^{\prime}$ are two homomorphisms of free groups, $F$ is finitely generated, and $\varphi$ is injective, then $\operatorname{Eq}(\varphi, \phi)$ is also finitely generated. Note that this conjecture is stronger than the Scott conjecture since, if $\varphi$ is an isomorphism, then $\operatorname{Eq}(\varphi, \phi)=\operatorname{Fix} \phi \varphi^{-1}$. Note also that this statement is easily seen to be false when both morphisms have non-trivial kernel. Consider the free group $F_{2}=\langle a, b\rangle$ and the two endomorphisms $\varphi, \phi$ given by $a \varphi=1, b \varphi=b$ and $a \phi=1, b \phi=b^{-1}$; clearly, the equalizer $\operatorname{Eq}(\varphi, \phi)$ is the normal closure of $a$ in $F_{2}$, which has infinite rank. In [22], another example for this fact is produced but with the common image of the equalizer being not finitely generated.

Assuming that $\varphi$ must be injective, one can restrict the attention to the case where $F$ is a subgroup of $F^{\prime}$ and $\varphi$ is the inclusion. Then, $\operatorname{Eq}(\varphi, \phi)=\operatorname{Fix} \phi$, and $\phi: F \rightarrow F^{\prime}$ is an arbitrary homomorphism. This setting is more general than working with endomorphisms of $F^{\prime}$, because the existence of an extension of $\phi$ to an endomorphism of the whole of $F^{\prime}$ is not required.

Similarly to the case of fixed subgroups, one can also ask (or conjecture) whether the rank of the equalizer of $\varphi, \phi: F \rightarrow F^{\prime}$ is bounded above by that of $F$, when $\varphi$ is injective. Or even more, one can ask if $r(\mathrm{Eq}(S)) \leq r(F)$ for every set $S$ of morphisms from $F$ to $F^{\prime}$ containing at least one injective morphism. As we will see below, the Stallings conjecture was proved some years later, while these stronger questions are still open today. We will generically refer to all of them as the equalizer conjecture.

At the 1983 AMS Summer meeting, J. Stallings pointed out that the graph constructed and used by S. Gersten to prove the finiteness of the rank of 1-autofixed subgroups of $F_{n}$, could also be constructed using the families of surfaces described by the 3-dimensional model constructed by Whitehead in the 1930's (see [54] and [55]). R. Goldstein and E. Turner developed this idea in a series of two papers, $[\mathbf{2 4}]$ and $[\mathbf{2 5}]$. As is said in the introduction to the first paper, it so happens 
that the use of three dimensions provides a freedom that makes it easier to describe and construct Gersten's graph, and to prove its main properties. In the first of these two papers, published in 1984, they gave an alternative proof for Gersten's Theorem and they improved Gersten's upper bound for the rank of the fixed subgroup of a given automorphism of $F_{n}$. In the second one, which appeared in 1985, they went further using the same techniques and proved the Stallings conjecture for the case of two injective homomorphisms.

Furthermore, in 1986, another paper by R. Goldstein and E. Turner appeared, [26]. Here, the authors improved their previous result, solving completely the Stallings conjecture:

ThEOREM 3.2 (Goldstein-Turner, [26]). Let $F$ and $F^{\prime}$ be two free groups with $F$ finitely generated. If $\varphi, \phi: F \rightarrow F^{\prime}$ are two morphisms and $\varphi$ is injective then $\operatorname{Eq}(\varphi, \phi)$ is finitely generated.

In this case, the proof is quite simple, short and independent of the previous ones, and it proves a stronger result. In the situation where $F$ is a subgroup of $F^{\prime}$ and $\varphi$ is the inclusion, the authors provide a particular description of the covering space $X$ of a bouquet corresponding to the inclusion Fix $\phi \leq F$. Then, they choose an appropriate orientation on the edges of $X$ and use it to show that this graph has finite rank, and then so does Fix $\phi$.

In 1987, D. Cooper published [10] with another proof of Gersten's Theorem. This time, the methods used are of a topological and dynamical nature. They are inspired by work of W. Thurston about surface groups. The idea was to extend a given automorphism of $F_{n}$ to a homeomorphism of its end completion $\hat{F}_{n}$, a compact metric space where $F_{n}$ is dense. The author then showed that the fixed point set of this extension is finitely generated in a certain topological sense, which implies the finite generation of the subgroup fixed by the original automorphism.

In part inspired by D. Cooper's work, three more papers appeared, developing new concepts and results involving infinite words. These works made it clear that a close connection between the apparently different proofs of $[\mathbf{1 0}]$ and $[\mathbf{2 6}]$ exists. Two of them are [31] and [32], both published in 1990. In [32], W. Imrich and E. Turner considered an arbitrary homomorphism $\phi: F \rightarrow F^{\prime}$ from a subgroup $F$ of an arbitrary free group $F^{\prime}$, to $F^{\prime}$. By extending Goldstein-Turner techniques, they obtained new upper bounds for the rank of Fix $\phi$, and for the rank of the fixed point set (à la Cooper) of an injective $\phi$. These bounds are expressed in terms of a Nielsen reduced basis for $F$.

The third paper mentioned above is [6], by M.M. Cohen and M. Lustig, and published in 1989. In this interesting work, the authors extended the GoldsteinTurner method by imposing a vector field (i.e. a preferred orientation of the edges of the first barycentric subdivision) on the graph constructed in [26]. They introduced the concepts of attractive edges, repulsive edges, attracting fixed infinite words and attracting fixed points at infinity, and investigated the resulting dynamics.

In [6], the authors gave two explicit formulas for the exact value of the rank of the fixed subgroup of a given automorphism of $F_{n}$, one in geometric terms and the other in a completely algebraic context. In general, it is not easy to make use of these formulas for concrete examples and, also, they do not seem to give enough information to derive a uniform upper bound for the rank of the 1-autofixed subgroups of $F_{n}$ depending only on $n$. However, they applied their method 
to obtain an explicit algorithm for computing the fixed subgroup of a positive automorphism of $F_{n}$. Recall that a positive automorphism is one for which there exists a basis of $F_{n}$ whose images are positive words, see section 6 in [6]. They also obtained some useful results of which the following are a sample:

ThEOREM 3.3 (Cohen-Lustig, [6]). Let $\left\{x_{1}, \ldots, x_{n}\right\}$ be a basis of the free group $F_{n}$, and let $\phi \in A u t\left(F_{n}\right)$. If $r_{i}$ denotes the number of occurrences of $x_{i}$ in (the normal form of ) $x_{i} \phi$ of the form $x_{i} \phi=v \cdot x_{i} \cdot w$, with $v, w \in F_{n}$ and the abelianization of $w$ belonging to the image of $I d-\phi^{\mathrm{ab}}$, then $r(\mathrm{Fix} \phi) \leq \sum_{i=1}^{n} r_{i}$. In particular, if for every $i=1, \ldots, n, x_{i}$ does not occur in $x_{i} \phi$, then Fix $\phi=1$.

TheOREM 3.4 (Cohen-Lustig, [6]). Let $\left\{x_{1}, \ldots, x_{n}\right\}$ be a basis of the free group $F_{n}$, and let $\phi \in \operatorname{Aut}\left(F_{n}\right)$ be an automorphism such that $x_{1} \phi, \ldots, x_{n} \phi$ are positive words. If no word $x_{i} \phi$ begins or ends with $x_{i}$, then Fix $\phi=1$.

THEOREM 3.5 (Cohen-Lustig, $[\mathbf{6}])$. Let $\Phi \in \operatorname{Out}\left(F_{n}\right)$ be an outer automorphism of $F_{n}$. Then, $r($ Fix $\phi) \leq 1$ for every $\phi \in \Phi$ except for those belonging to a finite number of conjugacy classes of elements in $\Phi$.

There is also another extension of Gersten's Theorem in a different direction, due to J. Stallings in 1987. The paper [46], developed simultaneously to [25] of Goldstein-Turner, provided a proof for the following variation of the Stallings conjecture:

TheOREM 3.6 (Stallings, [46]). Let $F$ and $F^{\prime}$ be two free groups with $F$ finitely generated. Let $\varphi, \phi: F \rightarrow F^{\prime}$ be two homomorphisms with $\operatorname{ker} \varphi=\operatorname{ker} \phi$. Then the (common) image of $\operatorname{Eq}(\varphi, \phi)$ under $\varphi$ or $\phi$ is finitely generated. In particular, if $\varphi$ and $\phi$ are injective then $\operatorname{Eq}(\varphi, \phi)$ is finitely generated.

Here, J. Stallings developed his dyads and the graphic techniques of folds and ladder attachments. He used these techniques to build an extension of Gersten's proof suitable to study the more general situation treated in his paper. So, [46] focuses on the graphic and combinatorial point of view, without involving either 3dimensional arguments or infinite words (although it contains an appendix relating the dyads to Heegaard diagrams in 3-manifolds).

Additionally, [46] contains a list of the main questions and conjectures about this topic at that time. This list contains the above mentioned questions about $n$ being a uniform upper bound for the rank of 1-auto-fixed, 1-endo-fixed, auto-fixed or endo-fixed subgroups of $F_{n}$, or the corresponding ones for the equalizer of a family of two or more homomorphisms (one of them being injective). There is also another interesting question relating these problems with subgroups of $F_{n} \times F_{n}$.

Furthermore, one finds the papers [27] and [28], published by A.H.M. Hoare in 1988 and 1990, respectively. Here, the Whitehead 3-dimensional model ([54] and [55]), Gersten's graph ([22]), the Goldstein-Turner construction, $([\mathbf{2 4}],[\mathbf{2 5}])$ and the dyads of Stallings $([\mathbf{4 6}])$ are considered and related to each other. At the same time, the author offered some variations and extensions of all these constructions, as well as a combinatorial proof for the classical Whitehead cut vertex lemma using them. However, no new results about fixed subgroups were given.

It is also worth mentioning the extremely short paper [48] published by S. Thomas in 1988. Using previously known results from A.G. Howson, J. Stallings 
and J. McCool, as well as Gersten's Theorem, the author definitely closed the original question of Dyer-Scott in [15] by dropping the hypothesis on $G$ in Theorem 3.1:

TheOREM 3.7 (Thomas, [48]). Let $G$ be an arbitrary group of automorphisms of $F_{n}$. Then, Fix $G$ is finitely generated.

\section{The work of Bestvina-Handel}

The next step in this history is the deepest and most relevant one in the whole line of research about fixed subgroups in free groups. In $1988 \mathrm{M}$. Bestvina and M. Handel announced a proof for the stronger version of the Scott conjecture, namely the upper bound $n$ for the rank of 1-auto-fixed subgroups of $F_{n}$ :

Theorem 4.1 (Bestvina-Handel, [2]). Let $\phi$ be an automorphism of $F_{n}$. Then, $r(\operatorname{Fix} \phi) \leq n$.

This result provides the first (and the best possible) uniform upper bound for $r($ Fix $\phi)$, in contrast with the previously known ones, all of them being either not uniform or valid only for special families of automorphisms. This result appeared in published form, with a delay of almost four years, in the fifty-page paper [2] in the Annals of Mathematics in 1992. Again, some applications and improvements of the Bestvina-Handel Theorem appeared in print before [2] was published.

This excellent and dense work developed a new and powerful graphic theory to represent arbitrary automorphisms of $F_{n}$, and ended up with a proof of Scott conjecture as a consequence. It has a highly topological flavor and it is notoriously hard to read. It was inspired in the classical work of W. Thurston on homeomorphism of surfaces.

W. Thurston, following a program started many years before by J. Nielsen, gave a classification of homeomorphisms of a compact surface $S$, up to isotopy. Furthermore, he constructed a particularly efficient representative for every isotopy class, that is, for every element of the mapping class group of the surface, $M C G(S)$. This representative can always be chosen to be either of finite order, or pseudoAnosov, or reducible (i.e. constructible in a simple way, from homeomorphisms of simpler surfaces, which are either of finite order or pseudo-Anosov). Thurston's work was first described in the preprint [49] in 1976, three years later in [16] (the proceedings of a seminar held at Orsay in 1978), and finally published twelve years later in [50]. See also [5] for an introduction, and [3] for an algorithmic proof of Thurston's Theorem, based on [2].

Mapping class groups of compact surfaces, $M C G(S)$, and outer automorphism groups of free groups, $\operatorname{Out}\left(F_{n}\right)$, are similar in many ways. In [2], M. Bestvina and M. Handel developed a project analogous to Thurston's, replacing surfaces by graphs, and homeomorphisms by homotopy equivalences, a sort of one-dimensional version of Thurston's work. Their main result was the construction of a graph $Z$ and an efficient homotopy equivalence $\beta: Z \rightarrow Z$, inducing a previously given outer automorphism $\Phi \in \operatorname{Out}\left(F_{n}\right)$ at the fundamental group level. Here, efficiency consists of some good properties controlling the amount of cancellation appearing in the images of single edges of $Z$ under iterates of $\beta$.

One has to think of $F_{n}$ as the fundamental group of the bouquet $R_{n}$, a graph with a single vertex and $n$ edges. A marked graph is a connected graph $Z$ together with a homotopy equivalence $\rho: R_{n} \rightarrow Z$. Then, every homotopy equivalence 
$\beta: Z \rightarrow Z$ of a marked graph $Z$ induces an automorphism of $F_{n}$ up to conjugation, that is, an outer automorphism $\Phi \in \operatorname{Out}\left(F_{n}\right)$. It is then said that $\beta$ is a topological representative of $\Phi$, or that $\beta$ induces $\Phi$.

In the first part of [2], the authors analyzed the case of irreducible outer automorphisms. An outer automorphism $\Phi \in \operatorname{Out}\left(F_{n}\right)$ is said to be reducible if there exist free factors $G_{1}, \ldots, G_{k}$ of $F_{n}$ whose conjugacy classes are permuted by $\Phi$, and such that $G_{1} * \cdots * G_{k}$ is still a free factor of $F_{n}$; otherwise, it is called irreducible. See $[\mathbf{1 3}]$ for a classification of the simplest irreducible automorphisms. M. Bestvina and M. Handel proved that every irreducible automorphism can be topologically represented by a train track that is, a homotopy equivalence $\beta: Z \rightarrow Z$ such that $\beta^{k}$ is locally injective in the interior of every edge (i.e., no cancellation appears in the iterate images of every edge $e \beta^{k}$, for $e \in E Z$, and $k \geq 1$ ). Using these train tracks, they proved the following two results:

Theorem 4.2 (Bestvina-Handel, [2]). Let $\Phi \in$ Out $\left(F_{n}\right)$ be an irreducible outer automorphism of $F_{n}$. Then, $r($ Fix $\phi) \leq 1$ for every $\phi \in \Phi$.

Theorem 4.3 (Bestvina-Handel, [2]). Let $\Phi \in \operatorname{Out}\left(F_{n}\right)$ be such that $\Phi^{k}$ is irreducible for every $k \geq 1$. If there exist a cyclic word $s$ such that $s \Phi=s$ or $s \Phi=s^{-1}$, then $\Phi$ is geometrically realized by a pseudo-Anosov homeomorphism of a compact surface with one boundary component.

The former is a stronger version of the Scott conjecture for irreducible automorphisms. The latter was quickly used by S. Gersten and J. Stallings to solve the PV-conjecture (see $[\mathbf{2 3}]$ and the next section).

In section 5 of $[2]$, M. Bestvina and M. Handel extended their theory from irreducible to arbitrary automorphisms of $F_{n}$. Any homotopy equivalence $\beta: Z \rightarrow Z$ defines a natural filtration of $Z$ by (not necessarily connected) $\beta$-invariant subgraphs, $\emptyset=Z_{0}<Z_{1}<\cdots<Z_{r}=Z$, such that $Z_{i-1}$ is a maximal proper $\beta$-invariant subgraph of $Z_{i}, i=1, \ldots, r$. The subgraph $\operatorname{cl}\left(Z_{i} \backslash Z_{i-1}\right)$ is called the $i$-th stratum. It is proven that an outer automorphism is irreducible precisely when all its topological representatives over core graphs (i.e. finite graphs without vertices of valence one) containing no non-trivial $\beta$-invariant forests, contain no non-trivial $\beta$-invariant subgraphs. In this case, the corresponding filtration has only one stratum.

Now, they introduced the notion of relative train track which, up to technical details, is a natural relativization of the notion of train track, with respect to the previous filtration. With a considerable amount of technical work, the authors ended up with the result that every automorphism of $F_{n}$ admits a topological representative which is a relative train track. Then, analyzing Nielsen paths, i.e. paths in the graph fixed up to homotopy, they finally obtained the desired proof for the Scott conjecture.

The process of building such a good topological representative is controlled by a certain matrix. Here, the Perron-Frobenius Theorem (see Theorem 8.4.4 in [29] or II.1 in [14]) plays a central role. A square non-negative integer matrix $M$ is called irreducible when for every entry $(i, j)$ there exists $k \geq 0$ such that the $(i, j)$ th entry of $M^{k}$ is positive; otherwise, $M$ is reducible. Now, the Perron-Frobenius Theorem states that every irreducible matrix $M$ has the following properties: (i) it has a unique eigenvalue with maximum modulus, (ii) this eigenvalue is real, i.e. the 
spectral radius of $M$ is itself an eigenvalue, (iii) the corresponding eigenspace has dimension one, and (iv) this eigenspace is generated by a vector with all positive coordinates. Clearly, such a vector, when normalized, is uniquely determined by $M$ and it is called the Perron-Frobenius vector of $M$. Furthermore, if $M$ has integer entries, then its spectral radius cannot be a real number between 0 and 1 , i.e. it is 0,1 or larger than 1 .

It is easily seen that, for every square non-negative integer matrix $M$, there exists a permutation matrix $P$ such that $P^{-1} M P$ has the block form

$$
P^{-1} M P=\left(\begin{array}{cccc}
M_{1} & & & * \\
& M_{2} & & \\
& & \ddots & \\
0 & & & M_{r}
\end{array}\right)
$$

where $M_{1}, \ldots, M_{r}$ are irreducible submatrices, and the entries in the lower triangular part are zeros (note that $M$ is itself irreducible if and only if $r=1$ ). So, we can associate to $M$ the list of Perron-Frobenius eigenvalues of $M_{1}, \ldots, M_{r}$, say $\lambda_{1}, \ldots, \lambda_{r}$.

Let $\beta: Z \rightarrow Z$ be a homotopy equivalence. The transition matrix of $\beta$ is defined as the square non-negative integer matrix $M(\beta)$ whose entries are indexed by the set of edges in $Z$, and the $(i, j)$-th entry counts the number of times that the $\beta$-image of the $j$-th edge crosses the $i$-th edge (in either direction). Note that, in a suitable order of the edges of $Z$, the matrix $M(\beta)$ has the previous block form and this means that the subgraph $Z_{i}$ determined by the edges corresponding to $M_{1}, \ldots, M_{i}$ is $\beta$-invariant, $i=0, \ldots, r$. Also, the irreducibility of $M_{i}$ corresponds to the maximality of $Z_{i-1}$ as a $\beta$-invariant proper subgraph of $Z_{i}$. This defines the announced filtration $\emptyset=Z_{0}<Z_{1}<\cdots<Z_{r}=Z$. Additionally, the $i$-th stratum is called null, polynomial or exponential when the corresponding spectral radius $\lambda_{i}$ is 0,1 or bigger that 1 , respectively.

This construction allowed M. Bestvina and M. Handel to put a metric on the $i$-th stratum, by setting the length of every edge in $Z_{i} \backslash Z_{i-1}$ to be equal to the corresponding coordinate of the Perron-Frobenius vector of $M_{i}$. The useful property of such a metric is that, for every path $p$ in $Z_{i}$, the length of $p \beta^{k}$ is $\lambda_{i}^{k}$ times the length of $p$ (taking the length of the edges in $Z_{i-1}$ equal to zero). Hence, if $p$ is reduced, the difference between $\lambda_{i}^{k}$ times the length of $p$, and the length of the reduction of $p \beta^{k}$ measures the amount of cancellation occurring in $p \beta^{k}$. This, together with the Bounded Cancellation Lemma (see [2] for details) are the main tools for controlling cancellation.

The process of constructing a relative train track for $\Phi \in O u t\left(F_{n}\right)$ (and getting all the good technical properties) starts with a given topological representative of $\Phi$, for instance, the one over the bouquet. Then, M. Bestvina and M. Handel keep performing elementary operations (simple moves previously defined and analyzed) until they obtain a new representative of $\Phi$ with the desired properties. The key point is that none of these basic operations increases any of the Perron-Frobenius eigenvalues. This way, they control the whole process and are able to prove the existence of the desired representative, with the desired good technical properties.

Summarizing, an efficient representative $\beta: Z \rightarrow Z$ is constructed for any given automorphism $\phi \in \operatorname{Aut}\left(F_{n}\right)$ (in fact, $\beta$ represents the outer class $\Phi \in \operatorname{Out}\left(F_{n}\right)$ containing $\phi)$. The good technical properties of $\beta$ are enough to analyze how 
Nielsen paths intersect any given stratum. With this information, the authors construct a new graph $X$ and a map $X \rightarrow Z$ inducing the inclusion Fix $\phi \leq F_{n}$ at the fundamental group level. Since, by construction, any component of $X$ has rank at most $n$, they finally deduce that $r($ Fix $\phi) \leq n$, obtaining a proof of the Scott conjecture.

\section{Fixed subgroups after Bestvina-Handel}

Very soon after M. Bestvina and M. Handel announced their result in 1988, some applications, extensions and alternative proofs for the already proven Scott conjecture began to appear (some of them published even before [2]). The first one was [33], by W. Imrich and E. Turner, and published in 1989. Here, the authors extended Bestvina-Handel's result to arbitrary endomorphisms:

Theorem 5.1 (Imrich-Turner, [33]). Let $\phi \in \operatorname{End}\left(F_{n}\right)$ be an endomorphism of $F_{n}$. Then, $r(\operatorname{Fix} \phi) \leq n$.

In fact, they gave a simple algebraic argument to reduce the general situation to the bijective case, and then used Bestvina-Handel's result. Given $\phi \in \operatorname{End}\left(F_{n}\right)$, they considered the stable image $F_{n} \phi^{\infty}=\cap_{m=1}^{\infty} F_{n} \phi^{m}$. The key observation was that $F_{n} \phi^{\infty}$ is $\phi$-invariant, and that the restriction of $\phi$ to this subgroup is bijective. Since Fix $\phi \leq F_{n} \phi^{\infty}$ and $r\left(F_{n} \phi^{\infty}\right) \leq n$, the Bestvina-Handel Theorem applies to give the desired inequality, $r($ Fix $\phi) \leq n$.

In 1991, S. Gersten and J. Stallings published [23], where they used the special results in $[\mathbf{2}]$ which deal with the irreducible case, to solve the PV-conjecture. Precisely, they proved that every PV-automorphism is irreducible. Then, using Theorem 4.3 and the already known facts stating that any power of a PV-automorphism is again a PV-automorphism, and that PV-automorphisms are not geometric, they obtained the following result:

TheOREM 5.2 (Gersten-Stallings, $[\mathbf{2 3}]$ ). Every PV-automorphism of $F_{n}, n \geq 3$, has trivial fixed subgroup.

In 1994, D. Collins and E. Turner published [8]. Here, the authors reworked the whole Bestvina-Handel theory in a more general context, to obtain the same result for a larger class of groups. More precisely, they worked with free product groups, i.e. groups admitting a free decomposition into finitely many freely indecomposable factors. For those groups, there exists the notion of Kuroš rank, which is analogous to that of rank for free groups. Replacing graphs by graphs of complexes (i.e. graphs with several 2-complexes, called factor complexes, attached to certain vertices) it is also true that any such group $G$ is the fundamental group of a graph of complexes $\mathcal{Z}$, and that every automorphism of $G$ can be represented by a self-homotopy equivalence of $\mathcal{Z}$ which restricts to a homeomorphism on any factor complex. Mimicking the Bestvina-Handel theory, they built an efficient representative for any given automorphism of $G$, and they ended up with a proof of the corresponding Scott conjecture for automorphisms of free product groups (and with Kuroš rank in the place of the standard rank).

Another nice paper is [51], published in 1996 and dealing with retracts. A subgroup $H \leq F_{n}$ is called a retract of $F_{n}$ if the identity $I d_{H}$ extends to a morphism $r: F_{n} \rightarrow H$. Since, in this case, the composition of $r$ with the inclusion gives an 
idempotent endomorphism of $F_{n}$, called a retraction, retracts can alternatively be defined as images of retractions of $F_{n}$. Let $\phi \in \operatorname{End}\left(F_{n}\right)$ and let $H=F_{n} \phi^{\infty}$ be its stable image. In [51], E. Turner improved the result in [33] about stable images and, with a simple algebraic argument, proved that $H$ is always a retract. This had two new consequences relating fixed subgroups.

On the one hand, proper retracts of $F_{n}$ have rank strictly less that $n$, so we have:

TheOREM 5.3 (Turner, [51]). Let $\phi$ be an endomorphism of $F_{n}$. If $\phi$ is not bijective, then $r($ Fix $\phi) \leq n-1$.

In other words, 1-auto-fixed subgroups of $F_{n}$ with the maximal possible rank, $n$, can be fixed subgroups only of automorphisms. In particular, every 1-endo-fixed subgroup of $F_{n}$ having rank $n$ is 1-auto-fixed.

On the other hand, the fact that the stable image is a retract allows a connection with the concept of test word. A test word is an element $w \in F_{n}$ such that every endomorphism fixing $w$ is necessarily an automorphism. For example, the classical Nielsen commutator test says that $[a, b]=a^{-1} b^{-1} a b$ is a test word of $F_{2}=\langle a, b\rangle$ (see $[\mathbf{4 2}]$ ), and $\mathrm{H}$. Zieschang improved this by proving that in $F_{n}=\left\langle x_{1}, \ldots, x_{n}\right\rangle$, the words $x_{1}^{k} \cdots x_{n}^{k}$ for all $k \geq 2$, and $\left[x_{1}, x_{2}\right]\left[x_{3}, x_{4}\right] \cdots\left[x_{n-1}, x_{n}\right]$ when $n$ is even, are test words (see [56]). The connection with retracts found in [51] is the following:

ThEOREM 5.4 (Turner, [51]). A word $w \in F_{n}$ is a test word if and only if it is not contained in any proper retract of $F_{n}$.

As seen below, retracts are essential to understand the relation between the family of 1-endo-fixed subgroups of $F_{n}$, and the subfamily of 1-auto-fixed ones.

At this stage, the Scott conjecture was known to be true only for single endomorphisms. The next step in this direction was the corresponding result for families. This was proved for arbitrary families of injective endomorphisms by W. Dicks and E. Ventura in [14]:

THEOREM 5.5 (Dicks-Ventura [14]). Let $S$ be an arbitrary family of injective endomorphisms of $F_{n}$. Then, $r($ Fix $S) \leq n$.

In [14], this was proved as a corollary of the main result there, namely the inertia property for fixed subgroups of injective endomorphisms. We postpone the discussion of this and other related results to section 7 .

In $[43]$, Z. Sela gave another proof of this result using completely different methods. Motivated by Thurston's classification of automorphisms of a surface and the corresponding decomposition of such automorphisms, Z. Sela constructed in $[43]$ a hierarchical decomposition of a free group with respect to a given automorphism. His basic tool was a certain commutative diagram which establishes a link between algebraic invariants of automorphisms of hyperbolic groups (in particular free groups) and the dynamics of some associated actions on real trees. Using this commutative diagram, Z. Sela obtained Thurston's classification on the algebraic level, from a new point of view. The main technique involved is Rips' theory of stable actions of groups on real trees. As an application of his construction, Z. Sela deduced Theorem 5.5.

In 1998 two more related papers appeared. The recently developed theory of $\mathbf{R}$-trees provides powerful new techniques in combinatorial and geometric group 
theory. A nice application of these techniques was given by D. Gaboriau, G. Levitt and M. Lustig, who gave in [19] another proof for the Scott conjecture. This new proof is much shorter and simpler than Bestvina-Handel's, but, like Sela's proof, it is strongly based on the theory of group actions on real trees. The main result in [19] was the following. Given an outer automorphism $\Phi \in \operatorname{Out}\left(F_{n}\right)$, it is said that $\phi_{1}, \phi_{2} \in \Phi$ are similar when $\phi_{1}=c^{-1} \phi_{2} c$ for some inner automorphism $c$ of $F_{n}$. Observe that, in this event, Fix $\phi_{1}=\left(\text { Fix } \phi_{2}\right)^{c}$ so, these fixed subgroups have the same rank.

Theorem 5.6 (Gaboriau-Levitt-Lustig, $[\mathbf{1 9}])$. Let $\Phi \in \operatorname{Out}\left(F_{n}\right)$ be an outer automorphism of $F_{n}$, and let $\phi_{0}, \phi_{1}, \ldots, \phi_{k} \in \Phi$ belong to different similarity classes. Then,

$$
\sum_{i=0}^{k} \tilde{r}\left(\operatorname{Fix} \phi_{i}\right) \leq n-1
$$

In particular, $\Phi$ contains only finitely many similarity classes with a non-cyclic fixed subgroup.

As noted in [19], this theorem is only superficially stronger than 4.1. It follows by applying Theorem 4.1 to the automorphism of $F_{n} * F_{k}$ acting as $\phi_{0}$ on $F_{n}$ and sending the $i$-th generator $t_{i}$ of $F_{k}$ to $u_{i} t_{i}$, where $u_{i}$ is such that $\phi_{i}=\phi_{0} u_{i}$. Some authors use the term eigengroup of $\phi \in \operatorname{Aut}\left(F_{n}\right)$ with eigenvalue $u$ to refer to the fixed subgroup of $\phi u$ :

$$
\operatorname{Fix}(\phi u)=\left\{x \in F_{n}: u^{-1}(x \phi) u=x\right\}=\left\{x \in F_{n}: x \phi=u x u^{-1}\right\}
$$

(see [39] or Definitions I.5.5 in [14]). In this language, Theorem 5.6 can be stated by saying that the reduced ranks of the conjugacy classes of eigengroups of any given automorphism of $F_{n}$ add up to at most $n-1$.

In fact, the graph $X$ in the Bestvina-Handel construction is not connected in general and, as mentioned above, one of its components corresponds to the fixed subgroup of the automorphism under study. With a close look at section 6 in $[\mathbf{2}]$, one can conclude that the non-cyclic components of $X$ correspond exactly to the similarity classes of $\Phi$ with non-cyclic fixed subgroups. Hence, although not explicitly stated, the same argument in $[\mathbf{2}]$ shows Theorem 5.6.

The interpretation of Theorem 5.6 should read as follows: given $\phi \in \operatorname{Aut}\left(F_{n}\right)$, the bigger $r(\operatorname{Fix} \phi)$ is, the smaller $r(\operatorname{Fix}(\phi u))$ will be for every inner automorphism $u$ such that $\phi u \nsim \phi$. In the extremal case, if $r($ Fix $\phi)=n$ then every eigengroup of $\phi$ is either conjugate to Fix $\phi$ or cyclic.

Also in 1998, the paper [17] appeared, due to D. Gaboriau, A. Jaeger, G. Levitt and M. Lustig. This paper elaborates further on the techniques initiated in [19]. Actions on real trees are used to explore fixed infinite words or, more precisely, to relate the rank of the fixed subgroup with the number of fixed points at infinity. So, the results in $[\mathbf{1 7}]$ are in the line of those previously obtained by D. Cooper in [10], and by M. Cohen and M. Lustig in [6].

Let $\phi \in \operatorname{Aut}\left(F_{n}\right)$ be an automorphism of $F_{n}=\langle X\rangle, X=\left\{x_{1}, \ldots, x_{n}\right\}$. The boundary of $F_{n}$, denoted $\partial F_{n}$, is the set of reduced infinite words in $X^{ \pm 1}$, equipped with a natural topology, which makes $F_{n} \cup \partial F_{n}$ a compact topological space. Then, the automorphism $\phi: F_{n} \rightarrow F_{n}$ extends naturally to a homeomorphism $\phi: \partial F_{n} \rightarrow$ $\partial F_{n}$, and one can consider also the fixed points of this extension, that is, the so 
called infinite words fixed by $\phi$. Such an infinite word $W$ is called an attracting fixed point of $\phi$ when, for every $W^{\prime} \in F_{n} \cup \partial F_{n}$ close enough to $W$, one has $\lim _{p \rightarrow \infty} \phi^{p}\left(W^{\prime}\right)=W$. An algebraic version of this notion is the following (see [6]): the fixed infinite reduced word $W=a_{1} a_{2} \cdots$, with $a_{i} \in X^{ \pm 1}$, is attractive when for every $r>0$ there exist an integer $s_{0}$ such that for every $s \geq s_{0}, a_{1} a_{2} \cdots a_{s+r}$ is an initial segment of $\left(a_{1} a_{2} \cdots a_{s}\right) \phi$.

Two fixed infinite words $W_{1}$ and $W_{2}$ are said to be equivalent when $W_{2}=g W_{1}$ for some finite word $g \in$ Fix $\phi$. Note that any infinite word equivalent to an attracting fixed point of $\phi$ is itself an attracting fixed point of $\phi$. Then, let $a(\phi)$ denote the number of equivalence classes of attracting fixed points of $\phi$. The main result in $[\mathbf{1 7}]$ is the following improvement of Theorem 5.6, taking also into account the number $a(\phi)$ :

Theorem 5.7 (Gaboriau-Jaeger-Levitt-Lustig, [17]). Let $\Phi \in \operatorname{Out}\left(F_{n}\right)$ be an outer automorphism of $F_{n}$, and let $\phi_{0}, \phi_{1}, \ldots, \phi_{k} \in \Phi$ belonging to different similarity classes. Then,

$$
\sum_{i=0}^{k} \max \left(0, r\left(\operatorname{Fix} \phi_{i}\right)+\frac{a\left(\phi_{i}\right)}{2}-1\right) \leq n-1 .
$$

In particular, $\Phi$ contains only finitely many similarity classes with $r\left(\right.$ Fix $\left.\phi_{i}\right)+$ $\frac{a\left(\phi_{i}\right)}{2} \geq 2$.

The two main ingredients of the proof are the existence of a certain $\phi$-invariant R-tree, and an inequality by D. Gaboriau and G. Levitt about stabilizers of branch points in $\mathbf{R}$-trees (see [18]).

In 1999, A. Martino published [37], giving a similar result to Theorem 5.7 but for automorphisms of free product groups. This improved the result of CollinsTurner in [8] about the Scott conjecture for these groups, and provided an alternative proof of Theorem 5.7 for the free case. The arguments used are in the line of the Bestvina-Handel theory.

In 2000, O. Bogopolski published [4], where he considers the case of rank 2 and gives a list, up to change of basis, of all automorphisms of $F_{2}$ fixing a non-trivial element, together with an explicit description of the corresponding fixed subgroups. The paper also contains a complete list of the isomorphism types of the stabilizers of non-trivial elements of $F_{2}$ by the natural action of $\operatorname{Aut}\left(F_{2}\right)$.

Finally, it is worth mentioning the three recent preprints [35], [39] and [40].

In [35], M. Lustig solves the conjugacy problems in $\operatorname{Aut}\left(F_{n}\right)$ and $\operatorname{Out}\left(F_{n}\right)$. As a corollary to the algorithms given, he also provides an algorithm that, for any given $\phi \in \operatorname{Aut}\left(F_{n}\right)$, computes a basis for Fix $\phi$. So, the fixed subgroup of an automorphism of $F_{n}$ is algorithmically computable.

The main result in [39], due to A. Martino and E. Ventura, is an explicit description of what eigengroups of outer automorphisms of $F_{n}$ look like. The proof consists of closely analyzing the Bestvina-Handel construction at the fundamental group level. The version of this result concerning single automorphisms is the following:

THEOREM 5.8 (Martino-Ventura, $[\mathbf{3 9}]$ ). Let $F$ be a non-trivial finitely generated free group and let $\phi \in A u t(F)$ be an automorphism. Then, there exist integers $r, s \geq 0$, $\phi$-invariant subgroups $K_{1}, \ldots, K_{r} \leq F$, primitive elements $y_{1}, \ldots, y_{s} \in F$, 
a subgroup $L \leq F$, and elements $1 \neq h_{j}^{\prime} \in H_{j}=K_{1} * \cdots * K_{r} *\left\langle y_{1}, \ldots, y_{j}\right\rangle$, $j=0, \ldots, s-1$, such that

$$
F=K_{1} * \cdots * K_{r} *\left\langle y_{1}, \ldots, y_{s}\right\rangle * L
$$

and $y_{j} \phi=h_{j-1}^{\prime} y_{j}$ for $j=1, \ldots, s$; moreover,

$$
\text { Fix } \phi=\left\langle w_{1}, \ldots, w_{r}, y_{1}^{-1} h_{0} y_{1}, \ldots, y_{s}^{-1} h_{s-1} y_{s}\right\rangle
$$

for some non-proper powers $1 \neq w_{i} \in K_{i}$ and $1 \neq h_{j} \in H_{j}$ with $h_{j} \phi=h_{j}^{\prime} h_{j} h_{j}^{\prime-1}$, $i=1, \ldots, r, j=0, \ldots, s-1$.

Finally, the preprint [40], also by A. Martino and E. Ventura, analyzes the relationship between the families of 1-auto-fixed and 1-endo-fixed subgroups of $F_{n}$. It is easy to see that these two families coincide for $n=1,2$, but nothing was known for higher values of $n$. There, it is proved that the family of 1-endo-fixed subgroups of $F_{n}$ coincides with the family of 1-auto-fixed subgroups of retracts of $F_{n}$. Then, using Theorem 5.8, the first family of examples of retracts (and so 1-endo-fixed subgroups) of $F_{n}$ not being 1-auto-fixed is provided, for $n \geq 3$. One of the simplest such examples is the subgroup $\left\langle b, c a c b a b^{-1} c^{-1}\right\rangle$ of $F_{3}=\langle a, b, c\rangle$.

In the present section, the chronological order was not completely followed. Some papers were skipped and will be considered in the following two sections. In section 6 we will concentrate on the maximal rank case, which was studied for instance in the papers $[\mathbf{7}],[\mathbf{9}]$ and $[\mathbf{5 3}]$. And section 7 will be dedicated to the concept of inertia for fixed subgroups. This concept was first introduced in the monograph [14], and further used in $[\mathbf{1}]$ and $[\mathbf{3 8}]$.

\section{The maximal rank case}

Once it was known that 1-auto-fixed subgroups of $F_{n}$ have rank at most $n$, it was a natural question to ask for particular properties or descriptions of those subgroups with maximal rank. A 1-auto-fixed subgroup of $F_{n}$ having rank $n$ is called a maximum-rank 1-auto-fixed subgroup of $F_{n}$.

In 1993 the first paper studying this case appeared. It was [7], due to D. Collins and E. Turner. The authors analyzed the Bestvina-Handel theory in the case of maximal rank and obtained the result that any such subgroup contains a primitive element of $F_{n}$ :

THEOREM 6.1 (Collins-Turner, $[\mathbf{7}]$ ). Every automorphism of $F_{n}$ with fixed subgroup of rank $n$ fixes a primitive element of $F_{n}$.

A non-connected version of this result is given in section 3 of [19]. With the techniques developed there, D. Gaboriau, G. Levitt and M. Lustig proved that, if equality holds in (5.1), then some $\phi_{i}$ with $r\left(\right.$ Fix $\left.\phi_{i}\right) \geq 2$ fixes a primitive element of $F_{n}$.

Three years later, D. Collins and E. Turner published [9], where they went further in their project. By making a closer analysis of the Bestvina-Handel theory in the particular situation of maximal rank, the authors proved that in this case all the strata in the filtration of a relative train track must be polynomial, and they have to be of a very special type. They were able to read enough information from this graphic context to obtain the following very explicit algebraic description of 
maximum-rank 1-auto-fixed subgroups of $F_{n}$. Here, $F_{n}^{\text {ab }}$ denotes the abelianization of $F_{n}$, and for $a, b \in F_{n}$ we write $[a, b]=a^{-1} b^{-1} a b$.

TheOREM 6.2 (Collins-Turner, $[\mathbf{9}]$ ). Let $H$ be a subgroup of $F_{n}$, and let $m$ denote the rank of the (free abelian) image of $H$ in $F_{n}^{\mathrm{ab}}$ (called the abelian rank of $H)$. The following are equivalent:

a) $H$ is a maximum-rank 1-auto-fixed subgroup of $F_{n}$,

b) there exists a basis $\left\{x_{1}, \ldots, x_{n}\right\}$ of $F_{n}$ such that, setting $F_{l}=\left\langle x_{1}, \ldots, x_{l}\right\rangle$ for $0 \leq l \leq n$, there exists a basis $\left\{y_{1}, \ldots, y_{n}\right\}$ of $H$ such that for $1 \leq j \leq$ $m, y_{j}=x_{j}$, and for $m+1 \leq k \leq n, y_{k}=\left[w_{k}, x_{k}\right]$ for some $w_{k} \in H \cap F_{k-1}$ not being a proper power of any element of $F_{n}$ (so, in particular, $w_{k} \neq 1$ ).

In this event, $\left\{y_{1}, \ldots, y_{l}\right\}$ is a basis of $H \cap F_{l}$, for $0 \leq l \leq n$. Furthermore, every automorphism $\phi \in \operatorname{Aut}\left(F_{n}\right)$ with $H \leq \operatorname{Fix} \phi$ is of the form $x_{j} \mapsto x_{j}$ and $x_{k} \mapsto w_{k}^{r_{k}} x_{k}$ for some non-zero integers $r_{k}, 1 \leq j \leq m, m+1 \leq k \leq n$.

The typical example of a maximum-rank 1-auto-fixed subgroup is given by the following automorphism of $F_{2}=\langle a, b\rangle: a \mapsto a, b \mapsto a^{r} b$, where $r$ is an integer. Its fixed subgroup is $H=\langle a,[a, b]\rangle=\left\langle a, b^{-1} a b\right\rangle$, and $m=1$, except when $r=0$ (in which case it is the whole $\left.F_{2}\right)$. However, for $n \geq 3$, the fixed subgroup of an automorphism of the type described in the last paragraph of the previous theorem can be bigger than $H$. For example, let $r$ be a non-zero integer and consider the automorphism $\phi_{r}$ of $F_{3}=\langle a, b, c\rangle$ given by $a \mapsto a, b \mapsto a b, c \mapsto a^{r} c$. For $r \neq 1$ we have Fix $\phi_{r}=\left\langle a, b^{-1} a b, c^{-1} a c\right\rangle$, which has abelian rank $m=1$. But Fix $\phi_{1}$ is bigger since it also contains $b^{-1} c$, and has abelian rank $m=2$ (in fact, Fix $\left.\phi_{1}=\left\langle a, b^{-1} c, b^{-1} a b\right\rangle=\left\langle a, b^{-1} c, c^{-1} a c\right\rangle\right)$.

Additionally, in $[\mathbf{9}]$ there is also an analogous result describing the fixed subgroups of automorphisms of free product groups, with maximal Kuroš rank.

Finally, the paper [53], published in 1997 by E. Ventura, also considered the case of maximal rank. The main result in [53] was the following:

TheOREm 6.3 (Ventura, [53]). Among the strictly ascending chains of maximum-rank 1-auto-fixed subgroups of $F_{n}$, the maximum length is exactly $n$.

The proof uses the Collins-Turner description of maximum-rank 1-auto-fixed subgroups of $F_{n}$. Essentially, it is a graphic proof and it involves immersions and coverings of graphs, as well as Stallings foldings. Also, a simple graphic proof of an old result from M. Takahasi was provided. For the particular case $n=2$, and being $H$ a maximum-rank 1-auto-fixed subgroup of $F_{2}$, a description was given of all those subgroups $K \leq F_{2}$ satisfying $r(K)=r(H \cap K)=2$. The main consequence derived from this result was the following theorem, which is clearly only valid in the rank 2 case:

THEOREM 6.4 (Ventura, [53]). Let $S$ be a non-empty set of non-identity endomorphisms of $F_{2}$ such that $r($ Fix $S)=2$. Then, $S \subseteq \operatorname{Aut}\left(F_{2}\right)$, and Fix $S=$ $\left\langle a, b^{-1} a b\right\rangle$ for some basis $\{a, b\}$ of $F_{2}$, and Fix $S=$ Fix $\phi$ for each $\phi \in S$.

In particular, this implies that every group of automorphisms $G \leq A u t\left(F_{2}\right)$ with $r($ Fix $G)=2$ is either trivial or infinite cyclic. The paper $[\mathbf{5 3}]$ ends by showing that, in the rank 2 case, the families of 1-auto-fixed, 1-endo-fixed, auto-fixed and endo-fixed subgroups do coincide. As mentioned above, in [40] an example is given of a 1-endo-fixed not 1-auto-fixed subgroup of $F_{n}$, for $n \geq 3$. 
It is not known in general whether the families of 1-auto-fixed and auto-fixed (or 1-endo-fixed and endo-fixed) subgroups of $F_{n}$ coincide (see 8.9 and 8.10 below). However, the paper [38] (reviewed later) gave a positive solution to this problem in the maximal rank case, as a consequence of the main result there. The result is the following:

THEOREM 6.5 (Martino-Ventura, $[\mathbf{3 8}]$ ). Let $H \leq F_{n}$ be a subgroup of $F_{n}$ with $r(H)=n$. The following are equivalent:

a) $H$ is a 1-auto-fixed subgroup of $F_{n}$,

b) $H$ is a 1-mono-fixed subgroup of $F_{n}$,

c) $H$ is a 1-endo-fixed subgroup of $F_{n}$,

d) $H$ is an auto-fixed subgroup of $F_{n}$,

e) $H$ is a mono-fixed subgroup of $F_{n}$,

f) $H$ is an endo-fixed subgroup of $F_{n}$.

\section{Inertia and fixed subgroups}

In the monograph [14], published in 1996 by W. Dicks and E. Ventura, a new concept was introduced: inertia of subgroups of a free group. The main result in this work was the inertia property for mono-fixed subgroups of $F_{n}$, a stronger result than the Scott conjecture for those subgroups. This result is a partial solution for the unsolved equalizer conjecture. A natural particular case of that conjecture was the motivation to look at the notion of inertia.

The general equalizer conjecture says that the equalizer of two homomorphisms $\varphi, \phi: F_{m} \rightarrow F_{n}$, one of them being injective, has rank bounded by $m$. If, for example, $\varphi$ is injective then there is no lose of generality in assuming that $F_{m}$ is a subgroup of $F_{n}$ and $\varphi$ is the inclusion. In this case, we have only one homomorphism to consider, $\phi: F_{m} \rightarrow F_{n}, F_{m} \leq F_{n}$, and $\operatorname{Eq}(\varphi, \phi)=\operatorname{Fix} \phi$. So, the equalizer conjecture is the Scott conjecture generalized to partial endomorphisms, i.e. homomorphisms from a certain subgroup of $F_{n}$, to $F_{n}$.

The case $F_{m}=F_{n}$ is the Scott conjecture already solved by Bestvina-Handel. The simplest step to consider next is the case when $\phi: F_{m} \rightarrow F_{n}$ extends to an endomorphism $\tilde{\phi}$ of $F_{n}$; in this case, Fix $\phi=F_{m} \cap$ Fix $\tilde{\phi}$. We can reset this situation by taking $\phi \in \operatorname{End}\left(F_{n}\right)$, and an arbitrary subgroup $K \leq F_{n}$, and asking whether $r(K \cap$ Fix $\phi) \leq r(K)$. This was the original motivation for the following definition. A subgroup $H \leq F_{n}$ is called inert when $r(K \cap H) \leq r(K)$ for every subgroup $K \leq F_{n}$. Of course, taking $K=F_{n}$, if $H \leq F_{n}$ is inert then $r(H) \leq n$.

Easy examples of inert subgroups of $F_{n}$ are the cyclic ones, and the free factors of $F_{n}$. Not so obvious is the fact that every rank 2 subgroup of $F_{n}$ is inert. This is because of work by G. Tardos on the H. Neumann conjecture. In [47] this conjecture, i.e. $\tilde{r}(H \cap K) \leq \tilde{r}(H) \tilde{r}(K)$ for every $H, K \leq F_{n}$, was proved when one of the two subgroups involved has rank 2 . This is precisely the same as saying that rank two subgroups of $F_{n}$ are all inert.

The main result in $[\mathbf{1 4}]$ provided new examples of inert subgroups of $F_{n}$ :

ThEOREM 7.1 (Dicks-Ventura, [14]). Every mono-fixed subgroup of $F_{n}$ is inert. In particular, $r($ Fix $S) \leq n$ for every $S \subseteq \operatorname{Inj}\left(F_{n}\right)$.

This result can be thought of both as the next step towards the solution of the general Scott conjecture (before, it was only known for single endomorphisms, 
and this was the first result for families), or as a partial solution for the general equalizer conjecture.

With a simple observation one can see that the family of inert subgroups of $F_{n}$ is closed under arbitrary intersections (this is clear for finite intersections, and a standard argument on a descending chain of subgroups proves it in the infinite case, see Corollary I.4.13 in [14]). Then, it is enough to prove Theorem 7.1 for 1-mono-fixed subgroups. This is what it is done in [14].

The work [14] contains a complete reformulation and extension of the Bestvina-Handel theory, improving it in the following four directions.

Thought the paper [2], there is a topological flavor in many arguments (continuity, limits, density, Cantor sets, etc). However, one observes that this is not essential because the final result, as well as all the intermediate ones, are of a purely combinatorial and algebraic nature. One of the goals of [14] was to reformulate the entire theory in [2] in a complete algebraic setting, making transparent its algebraic nature. The only essential information that is carried by a path in a graph, is the reduced sequence of edges crossed by the path. So, it turns out to be more convenient to think of a graph just as a combinatorial object, and continuous maps between graphs as formal maps sending edges (and reduced paths) to reduced paths. A good way of modelling this is the categorical language of groupoids. Associated to any graph $Z$, one has the fundamental groupoid $\pi Z$, which must be thought of as the set of paths in $Z$ modulo reduction, and with concatenation, which is a partially defined operation. Then, continuous maps simply translate into groupoid morphisms. One of the advantages of this setting is that the notion of tightening in [2] disappears completely in the new language. This is because, when tightening a given path $p$ in a graph, one obtains another path $p^{\prime}$, but they both give exactly the same element of $\pi Z$. Another advantage is that the fundamental group of $Z$ at a vertex $v$ is now viewed as a subgroup of the groupoid $\pi Z$. So, passing to the fundamental group does not involve a move to a different context, it is just the restriction to a certain subgroup. This way, continuous maps and what they induce at the fundamental group level are unified in a common context. Also, the notion of topological representability can just be expressed as a simple equivalence relation in the context of groupoid morphisms.

Another important point of this reformulation of [2] is that it avoids talking about the invariant filtration $\emptyset=Z_{0}<Z_{1}<\cdots<Z_{r}=Z$. Following an idea of Gaboriau-Levitt-Lustig, the entire argument of [2] is rewritten just looking at the top stratum, and then arguing by induction on the reduced rank of the underlying (not necessarily connected) graph. This simplifies considerably the technical details, since one only has to work with a single invariant subgraph (instead of the whole filtration) and a single matrix and Perron-Frobenius eigenvalue and eigenvector (instead of one for each stratum in the filtration).

While translating into the algebraic setting, the technical details were arranged in such a way that the surjectivity property of the automorphism is finally used nowhere. Hence, the entire argument, and so the final conclusion, works for injective endomorphisms and not just for automorphisms of $F_{n}$. This is the first generalization provided. However, the construction is far from being valid in the presence of a non-trivial kernel. 
The fourth and main improvement is the generalization of the theory to provide a proof of the inertia for fixed subgroups. This was achieved by mixing the BestvinaHandel theory with the pull-back technique developed by J. Stallings in [45]. Given an injective endomorphism $\phi \in \operatorname{Inj}\left(F_{n}\right)$, the authors of [14] considered the graph $X$ and the map $X \rightarrow Z$ constructed in section 6 of [2], and inducing the inclusion Fix $\phi \leq F_{n}$ (see section 4). This is where M. Bestvina and M. Handel used the good graphical properties of this graph and graph map to show that $\tilde{r}(X) \leq \tilde{r}(Z)$ (and consequently, $r($ Fix $\phi) \leq n$ ). Instead, in [14] the authors consider another arbitrary finite connected core-graph $Y$ and map $Y \rightarrow Z$ (this represents an arbitrary different subgroup $K \leq F_{n}$ ). Then, they look at the pull-back of these two graphical maps (previously arranged to be immersions), say $W \rightarrow Z$. By [45], the latter immersion represents the intersection $K \cap$ Fix $\phi$. And the good technical properties were enough not only to show that $\tilde{r}(X) \leq \tilde{r}(Z)$, but also to prove that the inequality passes through the pull-back diagram. In this way, it was proved that $\tilde{r}(W) \leq \tilde{r}(Y)$, which means $r(K \cap$ Fix $\phi) \leq r(K)$ for an arbitrary $K \leq F_{n}$. This is precisely the inertia of the fixed subgroup.

In fact, in [14] the non-connected version of the previous result was proved. The analysis of the algebraic meaning of the different components of $W$ yields a final inequality involving the sum of reduced ranks of subgroups of the form $K^{y} \cap \operatorname{Fix}\left(\phi x^{-1}\right)$, where $x$ ranges over a set of representatives of Reidemeister classes of $\phi$, and, for every $x, y$ ranges over a set of double coset representatives of $K \backslash F_{n} / \operatorname{Fix}\left(\phi x^{-1}\right)$ (see Theorem IV.5.5 in [14] for the details). This non-connected version of inertia was codified using sets acted on by $F_{n}$.

Another interesting fact about [14] is that all the details left to the reader in [2] were meticulously verified in the new context.

Finally, another concept is introduced in [14] by relaxing the inertia condition. A subgroup $H \leq F_{n}$ is called compressed when $r(H) \leq r(K)$ for every other subgroup $H \leq K \leq F_{n}$. Clearly, inert subgroups are compressed, and it is easy to see that retracts are also compressed. It is not known whether compressed subgroups are inert. It is even unknown whether retracts are inert. These problems are related with some conjectures and open questions about fixed subgroups (see section 8).

After [14], two more papers appeared in the literature using, or building upon, the concept of inertia. The first is [1] , due to G. Bergman and published in 1999. Among other results, $[\mathbf{1}]$ contains the following theorem, which definitely solved the remaining piece of the general Scott conjecture:

Theorem 7.2 (Bergman, [1]). Let $S \subseteq \operatorname{End}\left(F_{n}\right)$ be an arbitrary family of endomorphism of $F_{n}$. Then, $r($ Fix $S) \leq n$.

So, the Scott conjecture was completely solved by 1999. However, a new problem came up naturally from [14]. In despite of Bergman's result, one can ask if Fix $\phi$ is inert when $\phi$ has non-trivial kernel. As far as we know, this is still an open problem today (see section 8 for additional comments).

Bergman's proof of Theorem 7.2 is based on a reduction to the injective case by using his main result:

Theorem 7.3 (Bergman, [1]). Let $G$ be a finitely generated torsion-free group, $L$ an arbitrary group, and consider the free product $G * L$ and the projection $\pi: G *$ 
$L \rightarrow G$. If $\sigma_{1}, \sigma_{2}: G \rightarrow G * L$ are two group-theoretic sections of $\pi$, then $\operatorname{Eq}\left(\sigma_{1}, \sigma_{2}\right)$ is a free factor of $G$.

The proof of this result used results of Stallings-Swan and a detailed analysis of supports of derivations. Also, at the end of $[\mathbf{1}]$, there is an interesting list of questions. Some of them will be discussed in the next section.

It is also interesting to remark that W. Dicks and M. Dunwoody gave a different and more general proof of Theorem 7.3. In [12], they proved exactly the same result, but without using the torsion-free hypothesis. This new proof makes use of the Almost Stability Theorem, of protrees and also of some new folding sequence techniques.

Another work relying on the inertia property is [38], published in 2000 by A. Martino and E. Ventura. Here it was conjectured that every auto-fixed subgroup is 1-auto-fixed. The authors were not able to prove this fact (which is still open today), but their main result provides support for this conjecture:

Theorem 7.4 (Martino-Ventura, [38]). Let $S \subseteq \operatorname{End}\left(F_{n}\right)$ and let $M$ be the submonoid of $\operatorname{End}\left(F_{n}\right)$ generated by $S$. Then, there exists $\phi \in M$ such that Fix $S$ is a free factor of Fix $\phi$.

The proof was completely algebraic, and considered first $S$ containing two elements, one of them injective, then $S \subseteq \operatorname{Inj}\left(F_{n}\right)$ and, finally, the general case using Bergman's result. Theorem 7.4 naturally raises the question of whether a free factor of a 1-endo-fixed subgroup is again 1-endo-fixed. This is obviously true when $n=2$ but it is not true for $n \geq 3$, and the following counterexample is provided in $[\mathbf{3 8}]$. By Theorem 6.2, the subgroup

$$
\langle a,[a, b],[a, c]\rangle=\langle a\rangle *\langle[a, b],[a, c]\rangle
$$

of $F_{3}=\langle a, b, c\rangle$ is 1-auto-fixed. But the authors proved that any endomorphism of $F_{3}$ fixing $[a, b]$ and $[a, c]$ is forced to fix $a$. So, $\langle[a, b],[a, c]\rangle$ is a free factor of a 1-auto-fixed subgroup of $F_{3}$, which is not even endo-fixed. The example can be easily generalized to an arbitrary $n \geq 3$.

As mentioned in section 6, Theorem 7.4 also has consequences for the maximal rank case (see Theorem 6.5).

The paper [38] also introduced the concept of auto-fixed closure. The autofixed closure of a subgroup $H \leq F_{n}$ is $H^{c}=\operatorname{Fix}\left(A u t_{H}\left(F_{n}\right)\right)$, where $A u t_{H}\left(F_{n}\right)$ is the subgroup of $A u t\left(F_{n}\right)$ of those automorphisms $\phi$ such that $H \leq$ Fix $\phi$. As in classical Galois theory, $H \leq H^{c}$ but the inequality can be strict. For example, the previous example can be used to say that the auto-fixed closure of $H=\langle[a, b],[a, c]\rangle$ in $F_{3}$ is $H^{c}=\langle a,[a, b],[a, c]\rangle$. In general, the relationship between any given subgroup $H$ of $F_{n}$, and its auto-fixed closure is quite obscure.

\section{Conjectures and open problems}

A list of interesting problems and conjectures that still remain open is given in this final section. We further add some comments and discussions on them. Some of the questions mentioned here are also asked and discussed in the last section of $[\mathbf{1 4}]$ and $[\mathbf{1}]$.

First of all, it is completely satisfactory that the general Scott conjecture, the original motivation for this line of research, is already completely solved. The work of Bestvina-Handel, Imrich-Turner, Dicks-Ventura and Bergman, shows that 
$r($ Fix $S) \leq n$ for any arbitrary set $S$ of endomorphisms of $F_{n}$. However, as usually happens in mathematics, even when the original problem is completely solved, the line of research is not exhausted because other interesting questions came up naturally from the developed work. For example, it is not known if fixed subgroups of endomorphisms of $F_{n}$ are necessarily inert.

CONJECTURe 8.1 (Inertia conjecture). Every endo-fixed subgroup of $F_{n}$ is inert. Equivalently, retracts of $F_{n}$ are inert.

Discussion. Let $S \subseteq \operatorname{End}\left(F_{n}\right)$. In [14] it is proved that Fix $S$ is inert when $S \subseteq \operatorname{Inj}\left(F_{n}\right)$. So, the open problem is about endomorphisms with non-trivial kernel. In this case, Bergman showed in $[\mathbf{1}]$ that $r($ Fix $S) \leq n$ for arbitrary $S$, giving supporting evidence for the conjecture.

The set of inert subgroups of $F_{n}$ is closed under arbitrary intersections. So, it is enough to consider 1-endo-fixed subgroups. Let $\phi \in \operatorname{End}\left(F_{n}\right)$. By a result of E. Turner [51] $\phi$ restricts to an isomorphism of the stable image $F_{n} \phi^{\infty}$, which is a retract of $F_{n}$ containing Fix $\phi$. So, Fix $\phi$ is inert as a subgroup of a retract of $F_{n}$. Hence, the conjecture is equivalent to saying that retracts are inert. There is a fairly explicit description of retracts of $F_{n}$ (see exercise 15 in section 3.2 of [36]), but this does not seem to help in solving this problem.

Question 8.2 (Bergman, [1]). Let $R, H \leq F_{n}$ be two finitely generated subgroups of $F_{n}$. Is it always true that if $R$ is a retract of $F_{n}$ then $R \cap H$ is a retract of $H$ ?

Discussion. The motivation for this question is that an affirmative answer would immediately give a proof for the inertia conjecture. Observe also that the analogous question for free factors is true, namely the intersection of a free factor $R$ of $F_{n}$ with any subgroup $H \leq F_{n}$ is a free factor of $H$.

Note that one of the results of Bergman in [1] is that an arbitrary intersection of retracts of $F_{n}$ is again a retract of $F_{n}$.

CONJECTURE 8.3 (Equalizer conjecture). If $S$ is a set of homomorphisms from $F_{m}$ to $F_{n}$, one of them being injective, then $r(\mathrm{Eq}(S)) \leq m$. Equivalently, $\operatorname{Eq}(S)$ is inert in $F_{m}$.

Discussion. By restricting the homomorphisms in $S$ to a given subgroup $K \leq$ $F_{m}$, we see that the two statements of the conjecture are in fact equivalent.

By a result of Goldstein-Turner $[\mathbf{2 6}], \mathrm{Eq}(S)$ is finitely generated when $S$ is finite. And by a result of Bergman [1] , if there is a homomorphism $\omega: F_{n} \rightarrow F_{m}$ such that, for every $\phi \in S, \phi \omega$ is the identity of $F_{m}$ then $\operatorname{Eq}(S)$ is a free factor of $F_{m}$. In particular, $r(\operatorname{Eq}(S)) \leq m$ in this case.

Using an injective element in $S$, we can restate the conjecture in the following way: "Let $F_{m} \leq F_{n}$ and let $S$ be an arbitrary set of morphisms from $F_{m}$ to $F_{n}$. Then, $r($ Fix $S) \leq m$ (equivalently, Fix $S$ is inert in $F_{m}$ )". With this new formulation, the result in [14] says that the conjecture is true when the homomorphisms in $S$ all extend to injective endomorphisms of $F_{n}$.

CONJECTURE 8.4 (Compressed-inert conjecture). Every compressed subgroup of $F_{n}$ is inert.

Discussion. First, let us note that, using the Schreier formula, the conjecture is clearly true for subgroups of $F_{n}$ of finite index. 
Since every retract of $F_{n}$ is compressed, but there are compressed subgroups which are not retracts, this conjecture is strictly stronger than the inertia conjecture. So a positive solution would imply that endo-fixed subgroups of $F_{n}$ are inert.

Let $F_{m}$ be a compressed subgroup of $F_{n}$, and let $S$ be an arbitrary family of homomorphisms from $F_{m}$ to $F_{n}$. In [52], the following is proved: if it is true that compressed subgroups are inert, then $\operatorname{Eq}(S)$ is inert in $F_{m}$. So, this conjecture seems to be of a similar level of difficulty as the equalizer conjecture. inert?

QUESTION 8.5. Is there an algorithm to decide if a given subgroup $H \leq F_{n}$ is

Discussion. In [53] a simple graphic algorithm is given to decide if a given subgroup $H \leq F_{n}$ is compressed. However, the algorithm seems to be unable to control subgroups not containing $H$.

QUESTION 8.6. Is there an algorithm to decide whether a given subgroup $H \leq$ $F_{n}$ is 1-auto-fixed and, in this case, find an automorphism fixing $H$ ? Analogous questions can be asked for 1-mono-fixed, 1-endo-fixed, auto-fixed, mono-fixed and endo-fixed subgroups.

Discussion. This question asks for an algorithm dual to the one provided by M. Lustig in [35]. The description of 1-auto-fixed subgroups provided by Theorem 5.8, although being quite explicit, does not seem to be good enough from the algorithmic point of view.

QUESTION 8.7. Is there any algebraic characterization of 1-auto-fixed subgroups of $F_{n}$ ? Similarly for the other five types of fixed subgroups.

Discussion. Collins-Turner gave in [9] such a characterization for the maximal rank case. The only result that is known in this direction for the general case is Theorem 5.8 due to A. Martino and E. Ventura.

It is known that every endo-fixed subgroup $H$ is pure (i.e. $x^{r} \in H$ implies $x \in H$ ) and inert. However, these conditions, although being quite restrictive, especially the second one, are not enough to characterize fixed subgroups.

QUESTION 8.8. Is there any algorithm to compute the auto-fixed closure $H^{c}$ of a given subgroup $H \leq F_{n}$ ?

Discussion. Given an automorphism $\phi$ of $F_{n}$, this question amounts to understanding which elements of $F_{n}$ are forced to be fixed by $\phi$, if some others are. This can happen even with independent elements (see the example in [38] mentioned in the previous section). It is not clear how these relationships work, nor it is understood how a subgroup $H$ of $F_{n}$ determines its auto-fixed closure $H^{c}$.

Conjecture 8.9 (Martino-Ventura, [38]). Every auto-fixed subgroup of $F_{n}$ is 1-auto-fixed.

Discussion. This was conjectured in [38], which gives much of the information known in support of this conjecture. There, it was proved that every auto-fixed (resp. mono-fixed, endo-fixed) subgroup of $F_{n}$ is a free factor of some 1-autofixed (resp. 1-mono-fixed, 1-endo-fixed) subgroup of $F_{n}$. However, an example was shown of a free factor of a 1-auto-fixed subgroup of $F_{n}$ with $n \geq 3$, which is not even endo-fixed. 
In particular, the conjecture is true for $F_{2}$, and for maximum-rank auto-fixed subgroups.

The same can conjectured with the prefix mono- or endo- instead of auto-.

QUESTION 8.10. What are the relationships (with respect to inclusion) between the families of 1-auto-fixed, 1-mono-fixed, 1-endo-fixed, auto-fixed, mono-fixed and endo-fixed subgroups of $F_{n}$ ?

Discussion. It is easy to see that these six families of subgroups of $F_{n}$ coincide when $n=2$. By Theorem 6.5, they also coincide in the maximal rank case.

In general, apart from the obvious inclusions between them, the only information known is that, for $n \geq 3$, the family of 1-endo-fixed subgroups strictly contains that of 1-auto-fixed ones (see [40]). In between, there is the family of 1-mono-fixed subgroups, which is not known to coincide with any of the previous families.

Conjecture 8.9 and the discussion there are closely related with this question.

Question 8.11. Let $F$ be a free group and $\phi \in \operatorname{End}(F)$. Is Fix $\phi$ inert in $F$ ?

Discussion. If $F$ is finitely generated, this question has already been considered, and coincides with the inertia conjecture above (see 8.1). The only new question asked here is about the infinitely generated case. The general Scott conjecture is vacuous in this case, since the inequality $r(H) \leq r(F)$ is immediate for every subgroup $H \leq F$. However, the inertia property changes the situation here. It is not a trivial question to ask if the fixed subgroup of an endomorphism of $F$ is inert in $F$. A similar question about equalizers can also be asked. Nothing is known in this direction.

\section{Acknowledgments}

The author is indebted to the Mathematics Department of the City College of New York for the hospitality received during the academic year 2000-2001, when this paper has been written. He is also indebted to J. Burillo for several suggestions improving the paper. The author gratefully acknowledges partial support by the DGI (Spain) through grant BFM2000-0354 and by the DGR (Generalitat de Catalunya) through grant 2001BEA1400176.

\section{References}

[1] G.M. Bergman, Supports of derivarions, free factorizations and ranks of fixed subgroups in free groups, Trans. Amer. Math. Soc., 351 (1999), 1531-1550.

[2] M. Bestvina, M. Handel, Train tracks and automorphisms of free groups, Ann. of Math., 135 (1992), 1-51.

[3] M. Bestvina, M. Handel, Train tracks for surface homeomorphisms, Topology, 34(1) (1995), 109-140.

[4] O. Bogopolski, Classification of automorphisms of the free group of rank 2 by ranks of fixedpoint subgroups, J. Group Theory, 3 (2000), 339-351.

[5] A.J. Casson, S.A. Bleiler, Automorphisms of surfaces after Nielsen and Thurston, Cambridge University Press, (1988).

[6] M.M. Cohen, M. Lustig, On the dynamics and the fixed subgroup of a free group automorphism, Invent. Math., 96 (1989), 613-638.

[7] D.J. Collins, E.C. Turner, An automorphism of a free group of finite rank with maximal rank fixed point subgroup fixes a primitive element, J. Pure Appl. Algebra, 88 (1993), 43-49. 
[8] D.J. Collins, E.C. Turner, Efficient representatives for automorphisms of free products, Michigan Math. J., 41 (1994), 443-464.

[9] D.J. Collins, E.C. Turner, All automorphisms of free groups with maximal rank fixed subgroups, Math. Proc. Cambridge Philos. Soc., 119 (1996), 615-630.

[10] D. Cooper, Automorphisms of free groups have finitely generated fixed point sets, J. Algebra, 111 (1987), 453-456.

[11] W. Dicks, M.J. Dunwoody, Groups acting on graphs, Advanced Studies in Mathematics 17, Cambridge University Press, (1989).

[12] W. Dicks, M.J. Dunwoody, On equalizers of sections, J. Algebra, 216 (1999), 20-39.

[13] W. Dicks, E. Ventura, Irreducible outer automorphisms of growth rate one, J. Pure Appl. Algebra, 88 (1993), 51-62.

[14] W. Dicks, E. Ventura, The group fixed by a family of injective endomorphism of a free group, Contemp. Math., 195 (1996), 1-81.

[15] J.L. Dyer, G.P. Scott, Periodic automorphisms of free groups, Comm. Alg., 3 (1975), 195-201.

[16] A. Fathi, F. Laudenbach, V. Poenaru et al., Travaux de Thurston sur les surfaces, Astérisque, 66-67, Soc. Math. France, Paris, 1979.

[17] D. Gaboriau, A. Jaeger, G. Levitt, M. Lustig, An index for counting fixed points of automorphisms of free groups, Duke Math. J., 93(3) (1998), 425-452.

[18] D. Gaboriau, G. Levitt, The rank of actions on R-trees, Ann. Sci. École Norm. Sup., 28(5) (1995), 549-570.

[19] D. Gaboriau, G. Levitt, M. Lustig, A dendrological proof of the Scott conjecture for automorphisms of free groups, Proc. Edinburgh Math. Soc., 41(2) (1998), 325-332.

[20] S.M. Gersten, On fixed points of certain automorphisms of free groups, Proc. London Math. Soc., 48 (1984), 72-90. Addendum: 49 (1984), 340-342.

[21] S.M. Gersten, On fixed points of automorphisms of finitely generated free groups, Bull. Amer. Math. Soc., 8 (1983), 451-454.

[22] S.M. Gersten, Fixed points of automorphisms of free groups, Adv. Math., 64 (1987), 51-85.

[23] S.M. Gersten, J.R. Stallings, Irreducible outer automorphisms of a free group, Proc. Amer. Math. Soc., 111 (1991), 309-314.

[24] R.Z. Goldstein, E.C. Turner, Automorphisms of free groups and their fixed points, Invent. Math., 78 (1984), 1-12.

[25] R.Z. Goldstein, E.C. Turner, Monomorphisms of finitely generated free groups have finitely generated equalizers, Invent. Math., 82 (1985), 283-289.

[26] R.Z. Goldstein, E.C. Turner, Fixed subgroups of homomorphisms of free groups, Bull. London Math. Soc., 18 (1986), 468-470.

[27] A.H.M. Hoare, On automorphisms of free groups I, J. London Math. Soc., 38 (1988), 277-285.

[28] A.H.M. Hoare, On automorphisms of free groups II, J. London Math. Soc., 42 (1990), 226236.

[29] R.A. Horn, C.R. Johnson, Matrix analysis, Cambridge University Press, Cambridge (1990).

[30] A.G. Howson, On the intersection of finitely generated free groups, J. London Math. Soc., 29 (1954), 428-434.

[31] W. Imrich, S. Krstić, E.C. Turner, On the rank of fixed point sets of automorphisms of free groups, NATO Adv. Sci. Inst. Ser. C Math. Phys. Sci., 301 (1990), 113-122.

[32] W. Imrich, E.C. Turner, Fixed subsets of homomorphisms of free groups, Topology and Combinatorial Group Theory, Lecture Notes in Math., 1440 (1990), 130-147.

[33] W. Imrich, E.C. Turner, Endomorphisms of free groups and their fixed points, Math. Proc. Cambridge Philos. Soc., 105 (1989), 421-422.

[34] W. Jaco, P.B. Shalen, Surface homeomorphisms and periodicity, Topology, 16 (1977), 347367.

[35] M. Lustig, Structure and conjugacy for automorphisms of free groups I, II, preprints.

[36] W. Magnus, A. Karrass, D. Solitar, Combinatorial Group Theory, Interscience Publishers, New York, (1966).

[37] A. Martino, An index theorem for automorphisms of free products, J. Group Theory, 2 (1999), 199-211.

[38] A. Martino, E. Ventura, On automorphism-fixed subgroups of a free group, J. Algebra, 230 (2000), 596-607.

[39] A. Martino, E. Ventura, A description of auto-fixed subgroups in the free group, preprint. 
[40] A. Martino, E. Ventura, Examples of retracts in $F_{n}$ that are not the fixed subgroup of any automorphism, preprint.

[41] J. Nielsen, Untersuchungen zur topologie der geschlossenen flächen II, Acta Math., 53 (1929), $1-76$.

[42] J. Nielsen, Die automorphismen der allgemeinen unendlichen gruppe mit zwei erzeugenden, Math. Ann., 78 (1918), 385-397.

[43] Z. Sela, The Nielsen-Thurston classification and automorphisms of a free group I, Duke Math. J., 84 (1996), 379-397.

[44] J.R. Stallings, Topologically unrealizable automorphisms of free groups, Proc. Amer. Math. Soc., 84 (1982), 21-24.

[45] J.R. Stallings, Topology of finite graphs, Invent. Math., 71 (1983), 551-565.

[46] J.R. Stallings, Graphical theory of automorphisms of free groups, Combinatorial group theory and topology, Ann. of Math. Stud. 111, Princeton University Press, 1987.

[47] G. Tardos, On the intersection of subgroups of a free group, Invent. Math., 108 (1992), 29-36.

[48] S. Thomas, Fixed points of automorphisms of finitely generated free groups, Proc. Amer. Math. Soc., 103 (1988), 333.

[49] W. Thurston, On the geometry and dynamics of diffeomorphisms of surfaces I, Preprint, 1976.

[50] W. Thurston, On the geometry and dynamics of diffeomorphisms of surfaces, Bull. Amer. Math. Soc., 19(2) (1988), 417-431.

[51] E.C. Turner, Test words for automorphisms of free groups, Bull. London Math. Soc., 28 (1996), 255-263.

[52] E. Ventura, Endomorfismes de grups lliures finitament generats (catalan), PhD Thesis, Universitat Autònoma de Barcelona, (1995).

[53] E. Ventura, On fixed subgroups of maximal rank, Comm. Algebra, 25 (1997), 3361-3375.

[54] J.H.C. Whitehead, On certain sets of elements in a free group, Proc. London Math. Soc., 41 (1936), 48-56.

[55] J.H.C. Whitehead, On equivalent sets of elements in a free group, Ann. of Math., 37 (1936), $782-800$.

[56] H. Zieschang, Über automorphismen ebener diskontinuierlicher gruppen, Math. Ann. 166 (1966), 148-167.

Dept. Mat. Apl. III, Univiversitat Politècnica de Catalunya, Barcelona, SPAin, and, Dept. of Math., City College of New York (Cuny), USA.

E-mail address: enric.ventura@upc.es 